\title{
Self-adaptive deployment of services in mobile environments: a study of the communication reliability on the host election algorithm
}

\author{
Gabriel Guerrero-Contreras ${ }^{1}$ (D) Sara Balderas-Díaz ${ }^{1}$ - Carlos Rodríguez-Domínguez ${ }^{1}$. \\ José Luis Garrido ${ }^{1}$. Aurora Valenzuela ${ }^{2}$
}

Received: 1 June 2016 / Accepted: 30 September 2016 / Published online: 13 October 2016

(C) Springer International Publishing Switzerland 2016

\begin{abstract}
Self-adaptive service deployment and replication are one of the possible approaches to address the changing computational conditions of mobile-based environments in order to ensure certain quality attributes of the system. The host election for the dynamic placement of service replicas is one of the main steps in the process to ensure the availability, reliability and performance in mobile distributed systems. The reliability of the host election algorithm is essential for the proper functioning of these systems. However, its distributed execution may be affected by the dynamic conditions of the mobile network. This paper presents an analysis of the reliability of an election algorithm on the basis of two different transmission protocols: TCP, a reliable protocol, and UDP, a non-reliable transmission protocol. The election algorithm is part of a self-adaptive architecture designed to address the availability of the services deployed in dynamic mobile network environments.
\end{abstract}

Gabriel Guerrero-Contreras

gjguerrero@ugr.es

Sara Balderas-Díaz

sarabd@ugr.es

Carlos Rodríguez-Domínguez

carlosrodriguez@ugr.es

José Luis Garrido

jgarrido@ugr.es

Aurora Valenzuela

agarach@ugr.es

1 Software Engineering Department, E.T.S.I.I.T, University of Granada, C/ Periodista Daniel Saucedo Aranda S/N, Granada, Spain

2 Department of Forensic Medicine, Toxicology and Physical Anthropology, Faculty of Medicine, University of Granada, Avenida de Madrid 11, 18012 Granada, Spain
Keywords Software architecture - Service oriented architecture (SOA) · Self-configuration · Election algorithm . Service availability $\cdot$ Reliability $\cdot$ Transmission control protocol (TCP) · User Datagram Protocol (UDP)

\section{Introduction}

The Service oriented architecture (SOA) proposes a modular distribution of the functionalities of a system through services (8). It provides the foundations to build an interoperable and scalable system thanks to the use of standard protocols and service composition. However, SOA itself is not sufficient to be able to operate in dynamic network environments (5), such as Ad hoc Networks (MANETs) (4). Such environments pose new features (e.g., energy constraints, dynamic network topologies, dynamic demand patterns, and heterogeneity, among others) which, if not correctly addressed, may have a significant impact on the quality attributes of the services (2).

For instance, the reliability and performance of distributed applications are critically conditioned by the placement of the services in the distributed system (16). However, the nature of MANETs requires mechanisms that allow to determine an adequate deployment of the services at run-time because of their changing network conditions. This represents a challenge for software architects, who must make suitable architectural design decisions to address these challenges.

As a result, self-adaptive architectures have been gaining importance in the research community (22). A self-adaptive architecture has been complemented with self-* features (i.e., self-healing, self-configuration, self-optimization, and selfprotection (14)), and it has the capability to reduce the impact of context changes in the quality attributes of the system at run-time. 
Fig. 1 A hypothetical scenario within the mobile forensic workspace case study

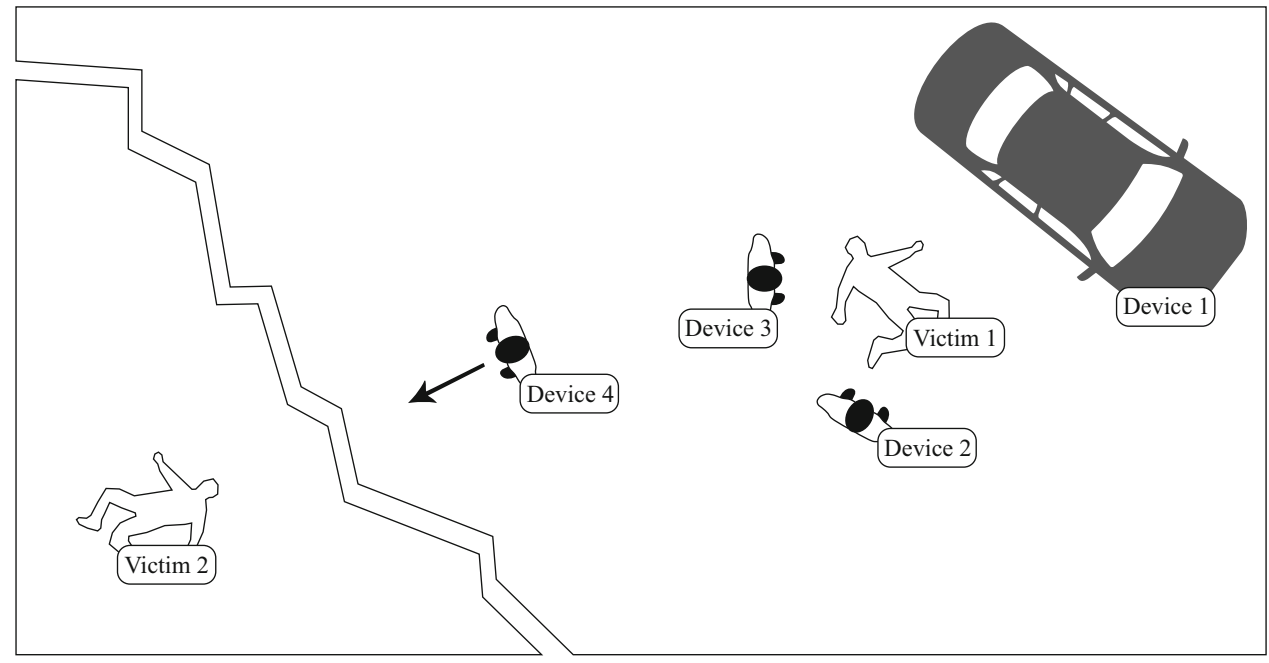

A self-adaptive software architecture has been proposed in $(9,11)$, which is intended to guarantee the availability of services in mobile collaborative environments. This software architecture consists of three main services: (1) the Monitoring Service, which is responsible for monitoring events in the context of the device that can affect the availability of a service; (2) the Context Manager Service, which stores information about the own device and other nodes of the network; and (3) the Replica Manager Service, which implements the election of the host device through a fully distributed election algorithm.

The aim of a host election algorithm (6) is to select the most suitable node (according to its hardware capabilities and the network status) in an ad hoc group of mobile nodes to act as a server (or coordinator) for the rest of the nodes of the group. The quality attributes of the service deployed will depend to a great extent on this election. Owing to the several issues that may influence the process (e.g., reliability and stability of the node, link status, users' behaviour, service nature, etc.), this would result in an NP complexity problem $(1,17)$. In this way, current approaches try to approximate the optimal solution.

The reliability of the host election algorithm is essential for the proper functioning of the self-adaptive architecture, and its distributed execution may be affected by the dynamic conditions of the mobile network. In this context, a reliable transmission protocol, as TCP, may be necessary. However, low latency, low overload of the operative system, and reduced data transmission of non-reliable transmission protocols, as UDP, are also important features for dynamic and resource constrained networks, as MANETs.

This paper presents a comparative study about the reliability of the host election algorithm proposed in (11) under a reliable transmission protocol (TCP) and a non-reliable transmission protocol (UDP). The aim is to analyse how TCP impacts on the reliability of the election algorithm, despite its high latency and higher consumption of bandwidth, in comparison to UDP. To this end, the software architecture has been implemented and simulated on the Network Simulator $3^{1}$.

The rest of this paper is organized as follows: Sect. 2 provides a motivating scenario for the self-adaptive architecture. Section 3 introduces a self-adaptive software architecture to support the dynamic replication and deployment of services. Section 4 presents the evaluation and results of the host election algorithm, under TCP and UDP transmission protocols, on the ns-3 network simulator. In Sect. 5, the results obtained are discussed. Finally, Sect. 6 draws some conclusions and outlines the plans for future research.

\section{Motivating scenario}

The Mobile Forensic Workspace (19), which allows forensic experts to exchange information in real-time for data sharing purposes in case of natural disasters, accidents, terrorist attacks, etc., is helpful to show the need and usefulness of the proposed architecture. The Fig. 1 depicts a hypothetical scenario where three members of a forensic team are gathering preliminary evidences of two victims. In the scenario two kinds of devices can be found: (1) a laptop located in a police car; and (2) three mobile devices (one per team member). Different functionalities provided by the corresponding services are required: direct voice communication between members, image repository, victim's information repository, etc. In the case of Image Repository service, each forensic expert can take several pictures of an evidence with his mobile device; different forensic experts can take addi-

\footnotetext{
${ }^{1}$ https://www.nsnam.org/.
} 
tional photos for the same evidence; or the same forensic expert can take other photos for evidences that belong to different victims. Moreover, the forensic experts can add annotations to these pictures, which can be related with other pictures. Therefore, the Image Repository service must keep an ordered and consistent set of this information, and at the same time it must provide high availability, to allow forensic experts to access and share pictures about the evidences found.

In this scenario, the proposed self-adaptive architecture is intended to improve the availability of the Image Repository service. This fact is highlighted in the following situations:

1. Initially, all forensic experts are near the Victim 1. All the mobile devices are connected with the laptop located in the police car. Therefore, the active service replica is the one deployed in the laptop (Device 1 in the Fig. 1), as it presents better computational features.

2. The forensic expert using the Device 4 will move to the area where the Victim 2 is located. This area is out of the laptop coverage. The self-adaptive architecture detects this situation and the adaptation process starts to carry out the deployment and activation of a new replica of the Image Repository service. As the only device available is itself, it will host an active replica of the service. At this point, the network is partitioned into two groups: (1) the Devices 1, 2 and 3, where the Device 1 hosts the active replica; and (2) the Device 4, in which the new service replica was deployed.

3. Later, the forensic experts of the Devices 2 and 3 move to the victim 2 area. At a given time the battery of the Device 4 is under the $25 \%$. The adaptation process will be started again to decide where to host a new service replica, with Device 2 and 3 as candidates. At this moment, there are no any clients using the service replica deployed in the laptop because of the network partition, therefore, that service replica will be hibernated in order to save resources.

\section{Self-adaptive software architecture}

The proposed software architecture has two main objectives: (1) provide a reusable and adaptable base for collaborative support systems, (2) enhance the availability of services in dynamic environments through an adaptive replication and deployment approach (9).

The architecture consist of the following five main services (Fig. 2):

- The Monitoring Service encompasses a set of monitoring services, which are sensing the context of the device in order to detect potential events that could affect the availability of a service. In the proposed architecture,

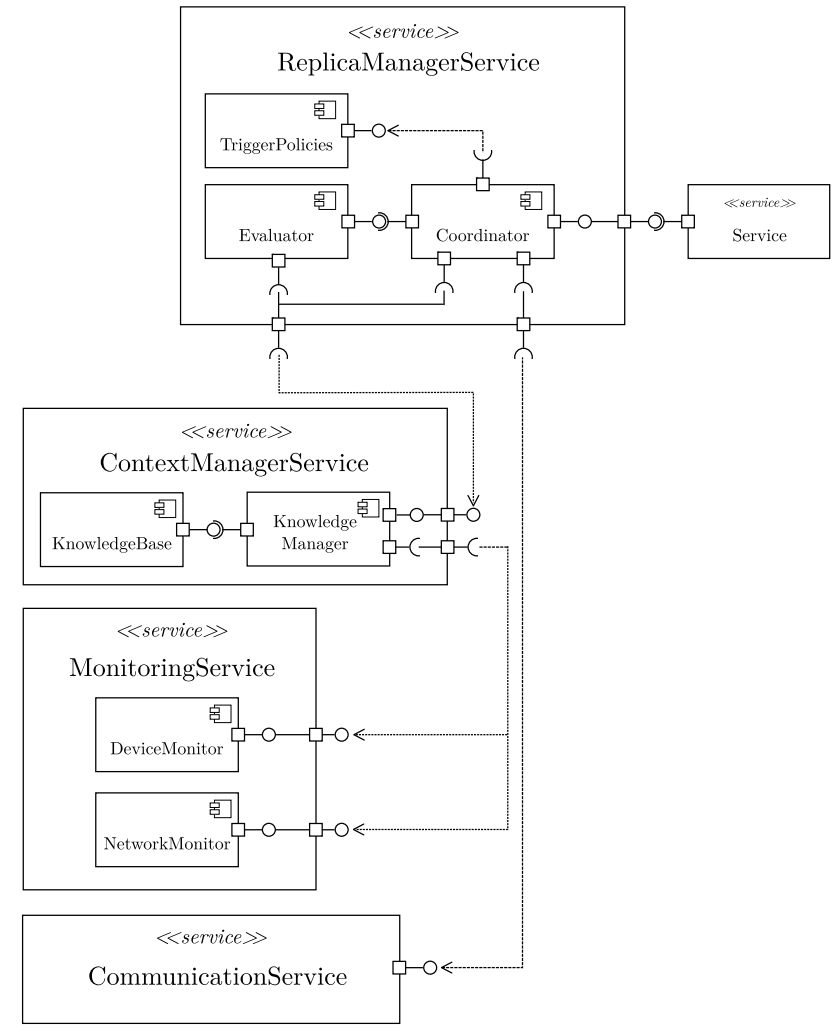

Fig. 2 Services and software components of the self-adaptive software architecture

device capabilities and network topology are monitored. The information about the device capabilities is local information that usually can be obtained easily, and the information provided by the routing protocol is used to estimate the network topology (11).

- The Context Manager Service is responsible for processing and storing the information received from the monitors of the device. This information will be used by the Replica Manager Service in order to adjust the deployment of the services according to the changes produced in the execution context. This information will be provided in two different ways: (1) under a Publish-Subscribe paradigm, where the Context Manager notifies, through a push-based communication model, the Replica Manager about events of its interest; and (2) under a RequestResponse paradigm, when, for example, the Replica Manager requests the Context Manager about information to evaluate the adequacy of the node. The combination of both paradigms is usually known as SOA 2.0 $(15,20)$ (a.k.a. advanced SOA), in which services are not just passive entities, but also they are able to receive and generate events proactively.

- The Communications Service, which allows the Replica Manager Service to communicate with other nodes of the network. 


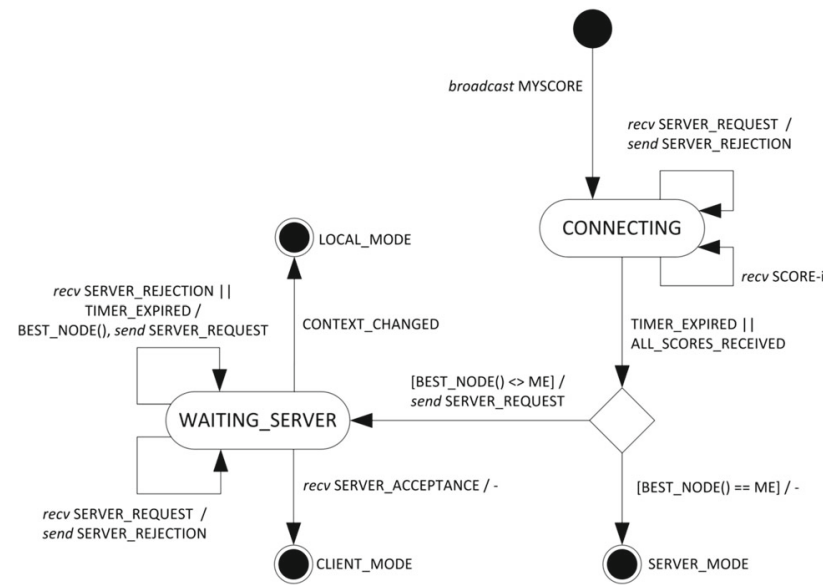

Fig. 3 The transition process between "Local Mode" state and the "Client Mode" or "Server Mode" states

- The Replica Manager Service encapsulates the adaptation logic regarding the replication and deployment of the service replicas. In order to provide a fully distributed solution, each service device has a Replica Manager Service replica, and this set of replicas are responsible for coming to an agreement to establish what will the active task service replica through the election algorithm. The host election algorithm follows a consensus approach and it can be initiated by any instance of the Replica Manager Service, as they have local information that is not accessible by the rest.

- the Service itself (i.e., the task application), which will be a passive or active replica according to the decision of the Replica Manager Service.

\subsection{Host election algorithm}

There are three possible states for each node: "Local Mode", "Client Mode" or "Server Mode". A node is in the "Local Mode" state when there is no reachable node in its neighbourhood. The availability of the service will be directly proportional to the time required for the transition between the "Local Mode" state and the new state ("Client Mode" or "Server Mode").

The adaptation process begins when there is some trigger policy that is activated by a change in the execution context or when the node receives the score of a neighbour, unleashing the adaptation. Each node acts according to the state diagram of the Fig. 3 (11), where the first step in the distribution host election process for a node is to calculate its score and broadcast it to its group. Then, the node goes into a passive mode where it waits to receive the scores of its neighbours. When the node receives all the scores of its neighbours, or the timer expires, the node calculates what is the most suitable node (within its group) to act as server.
In the calculation of the best node, the node will select the node of the group with the best score, this node could be itself. The score of the nodes is replicated information, this is, all the nodes within the group manage the same list of scores. Hence, all the nodes will take the same decision about what node will act as server, if there is no message lost. In the case of the best node will be the node itself, it will go into the "Server Mode" directly, assuming that the rest of the nodes will take the same decision. This approach avoids the server having to communicate its selection to the rest of the nodes, improving the use of the bandwidth. In the case where another node of the group is the best, the node will send a message requesting service to that node. At this point, one of the following situations can happen:

- The requested node is in the "Server Mode" state. Therefore, the requesting node will receive an affirmative answer and it will go to "Client Mode".

- The requested node is in the "Client Mode" state. The requesting node has not been sent the request to the best node of the group. This can be caused by two reasons: (1) the requesting node does not know all the nodes of the network yet, (2) some of the score messages have been lost. Thus, the requesting node has taken a wrong decision because it does not have all the information about the group. In this case, the requested node will respond with a rejection message. Additionally, this message will include information about the server of the requested node and its score, in order to complete the information of the requesting node.

- The requested node is still in transition. At this point, the requested node will return a rejection message, without additional information.

- The request message or the acceptance/rejection message is lost. In this case, once that the waiting timer has expired, the request will be made again.

Finally, in the case of the "Server Mode" state, when the node receives a request for service, it accepts the request through the server acceptance message. Note that not every node presents the necessary capabilities to operate as server. In this case, the node can only operate in the "Client Mode" or "Local Mode" states, and therefore it will not participate as candidate in the election, i.e., it will not evaluate itself or multicast its score.

\section{Evaluation}

\subsection{Simulation}

The proposed architecture has been simulated and evaluated using the ns-3 network simulator (Fig. 4), version 2.31, 
Fig. 4 Screenshot of one of the simulations performed with ns-3, obtained with the animator NetAnim ${ }^{4}$. In it is shown a network of ten nodes, which is divided into three partitions: a partition of seven nodes, where the Node 5 is the server; another of two nodes, where the Node 7 is the server; and the last, which is made up only by the Node 4, which is isolated

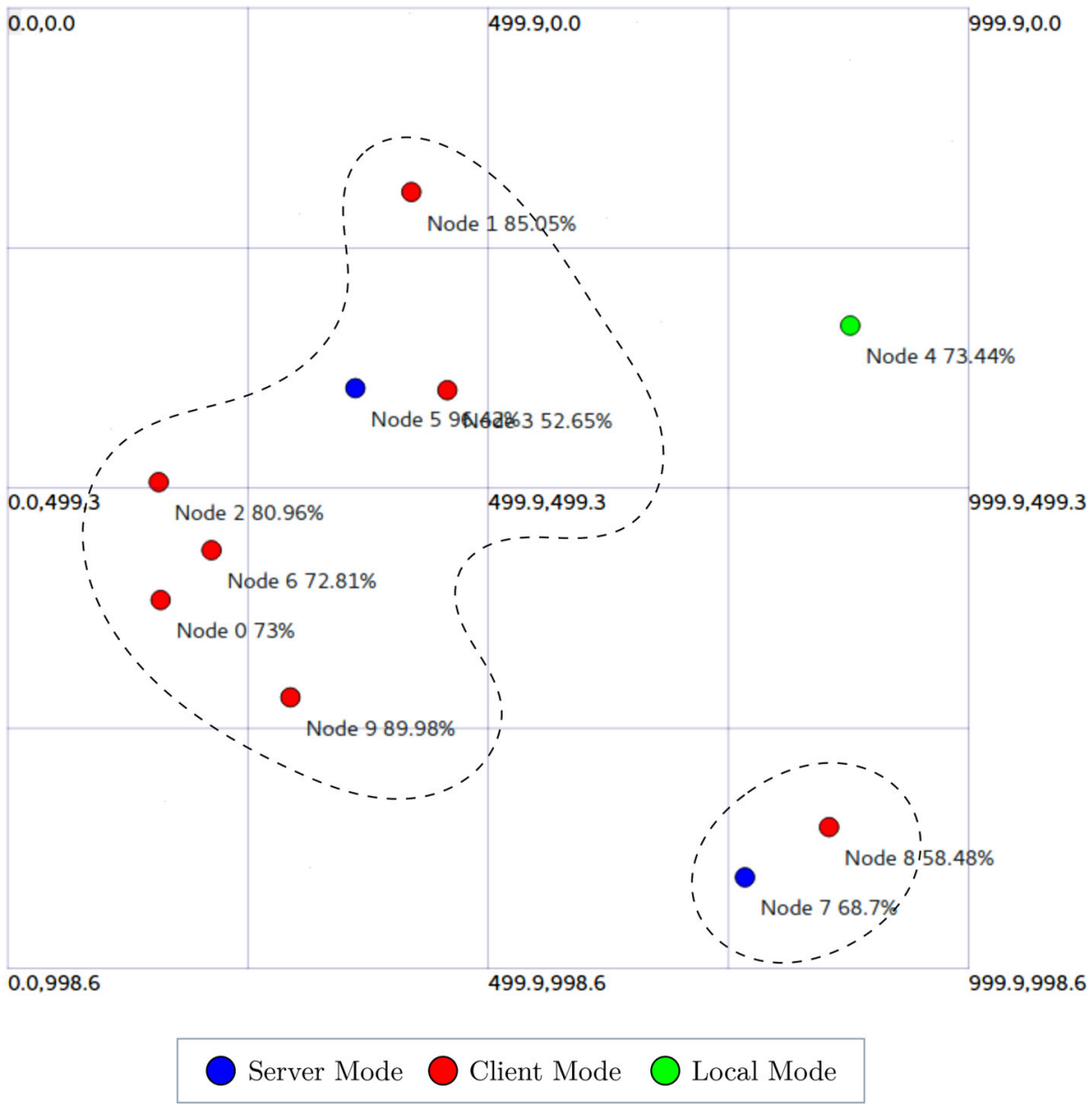

on a computer with an Intel Core i7-3630QM processor at $2.40 \mathrm{GHz}$ with 8 cores, $8 \mathrm{~GB}$ of memory and on Ubuntu 16.04 LTS 64-bit OS. The simulated scenario aims to approximate the situation of a work team, similar to the one described in Sect. 2, where different users are moving around of a wide scenario, sharing information through a mobile cloud service. It consists of a set of mobile nodes with a random walk mobility model. The speed of the nodes varies between $0.5-2 \mathrm{~m} / \mathrm{s}$, and they have pauses with a duration that varies between 60-300 s. The nodes have an IEEE 802.11 wireless connection, with a range of $250 \mathrm{~m}$ and a bandwidth of $1 \mathrm{Mbps}$. The mobility area is $1000 \mathrm{~m}^{2}$, and they are introduced in a random initial position. The time of the simulated execution is three hours. Each of the nodes has a Replica Manager Service, and a replica of the service. In this implementation, the "Optimized Link State Routing (OLSR) routing protocol has been used to make possible multi-hop communication between the nodes.

Under these conditions, two different versions of the host election algorithm has been implemented: (1) based on a TCP communication (reliable), and (2) based on a UDP communication (non-reliable). The number of nodes has ranged from 4 to 14 . Furthermore, in order to eliminate the influence of any possible random factor, the simulation has been performed 100 times with 100 different random seeds for each configuration.

\subsection{Reliability: definition and measurement}

The reliability of a system can be defined as "the ability of a system to perform as designed, with-out failure, in an operational environment, for a stated period of time." (21). Thus, in order to measure the reliability of a system, or usually, of a system component, it is firstly required to define when it fails.

In this work, we assume that the election algorithm fails when more than one node is designed to act as a server in the same group. This failure happens when some of the score messages from the most valuable node are lost. In such a case, the consensus approach followed by the nodes of the group fails, as not all the nodes have the same information (Sect. 3.1). For example, this situation is shown in Fig. 5. There is a group of four nodes with the following scores: A (0.8), B (0.6), C (0.7), and D (0.5) (Fig. 5a). If the score messages of 


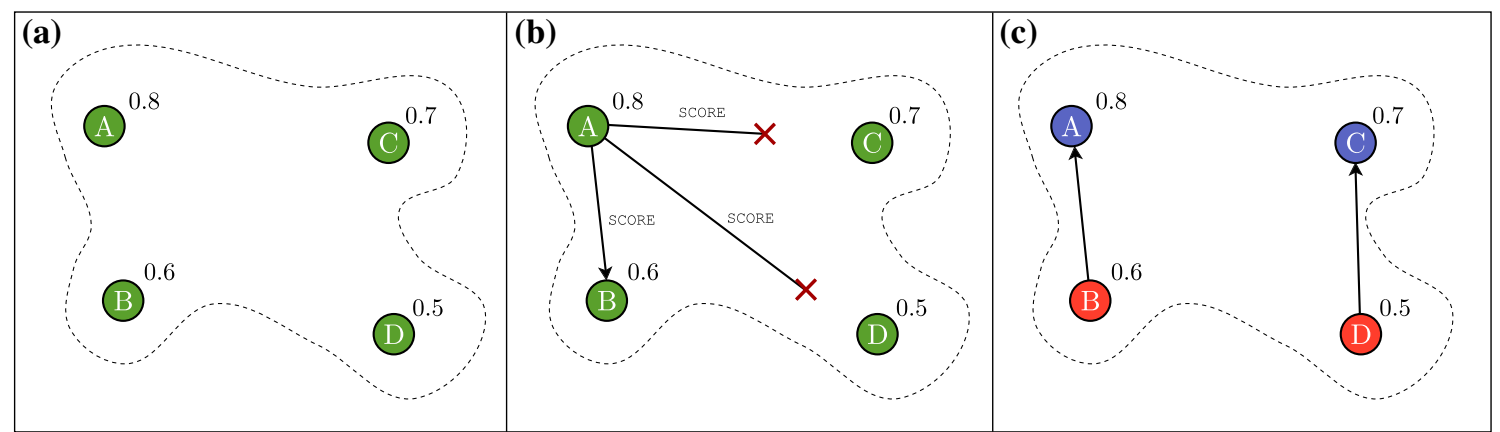

Fig. 5 Example situation where the loss of score messages leads to a fail of the consensus election algorithm group of the Fig. 5.c
Table 1 Score lists of the node

\begin{tabular}{lllll}
\hline & \multicolumn{4}{l}{ Score list } \\
\cline { 2 - 5 } Node & A & B & C & D \\
\hline A & $\mathbf{0 . 8}$ & 0.6 & 0.7 & 0.5 \\
B & $\mathbf{0 . 8}$ & 0.6 & 0.7 & 0.5 \\
C & - & 0.6 & $\mathbf{0 . 7}$ & 0.5 \\
D & - & 0.6 & $\mathbf{0 . 7}$ & 0.5 \\
\hline
\end{tabular}

the node $\mathrm{A}$ do not reach $\mathrm{C}$ and $\mathrm{D}$ (Fig. 5b), the score list of each node will be as is shown in Table 1 . Then, the nodes $\mathrm{A}$ and $\mathrm{B}$, will elect to node $\mathrm{A}$ as server, meanwhile the $\mathrm{D}$ and $\mathrm{C}$ will elect to node $C$ (Fig. 5c). This situation usually happens when the links within the nodes are not stable, which leads to a non-stable node group.

According to the previous definitions, two main measures to evaluate the reliability of the host election algorithm will be considered: (1) the percentage of failures according the total executions of the election algorithm during the simulation, (2) and the Mean Time Between Failures (MTBF) (21).

\subsection{Results}

The Fig. 6 shows the average failures in the execution of the host election algorithm. As shown, the increment of the size of the network leads to an increment of the average failures in the execution of the host election algorithm. This is caused by the increment of changes in the network topology, which leads to a less stable network. Both protocols increment the failures lineally according to the number of nodes of the network. However, the growth rate of UDP is faster than TCP. Regarding the use of bandwidth, TCP has only shown a slight increase in the traffic generated (of $0.0969 \mathrm{~KB}$ in average) with respect to UDP.

In the Fig. 7 is shown the average, maximum and minimum percentage of failures in the execution of the host election algorithm according to the total of executions. As can be seen from the Table 2, the election algorithm presents with TCP in average a $1.15 \%$ of average failures according to the total of executions of the host election algorithm. Whereas, with UDP, it presents in average a $2.00 \%$ of average failures.

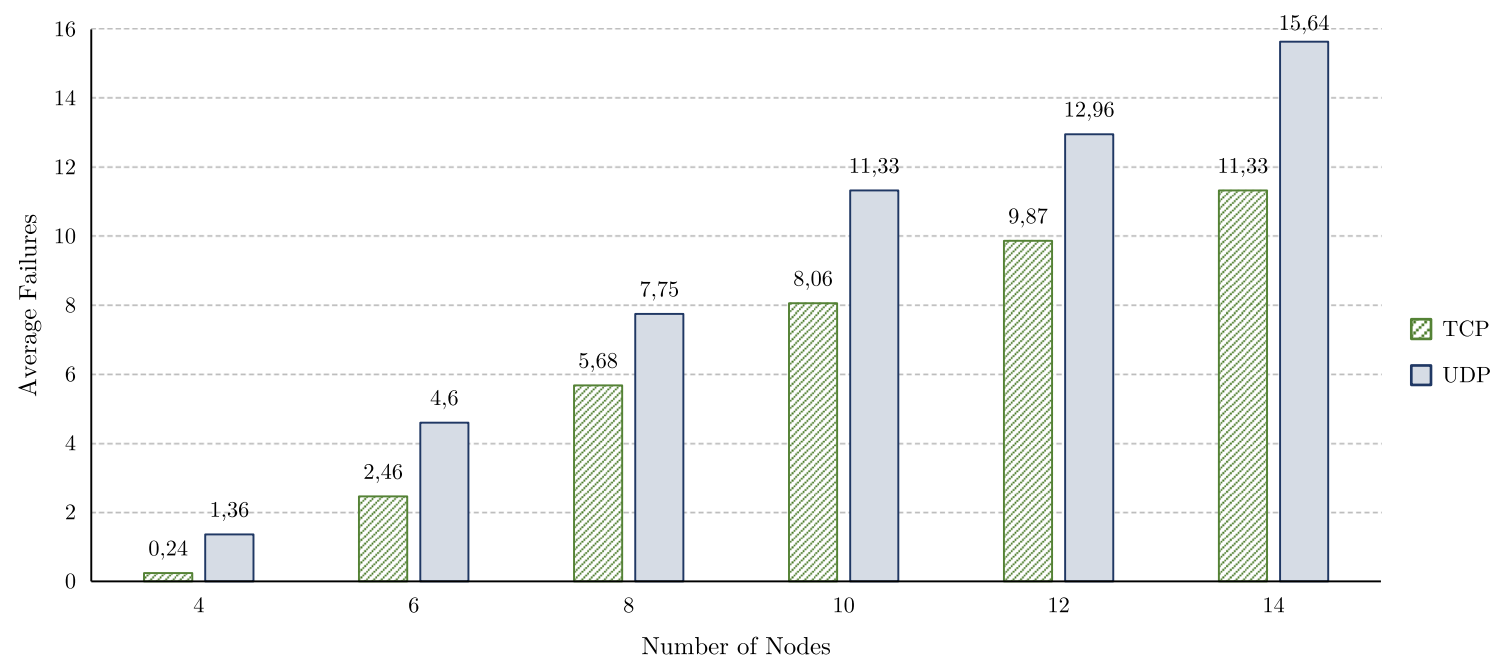

Fig. 6 Average failures of the host election algorithm, under TCP and UDP communication protocols, in networks from 4 to 14 nodes 

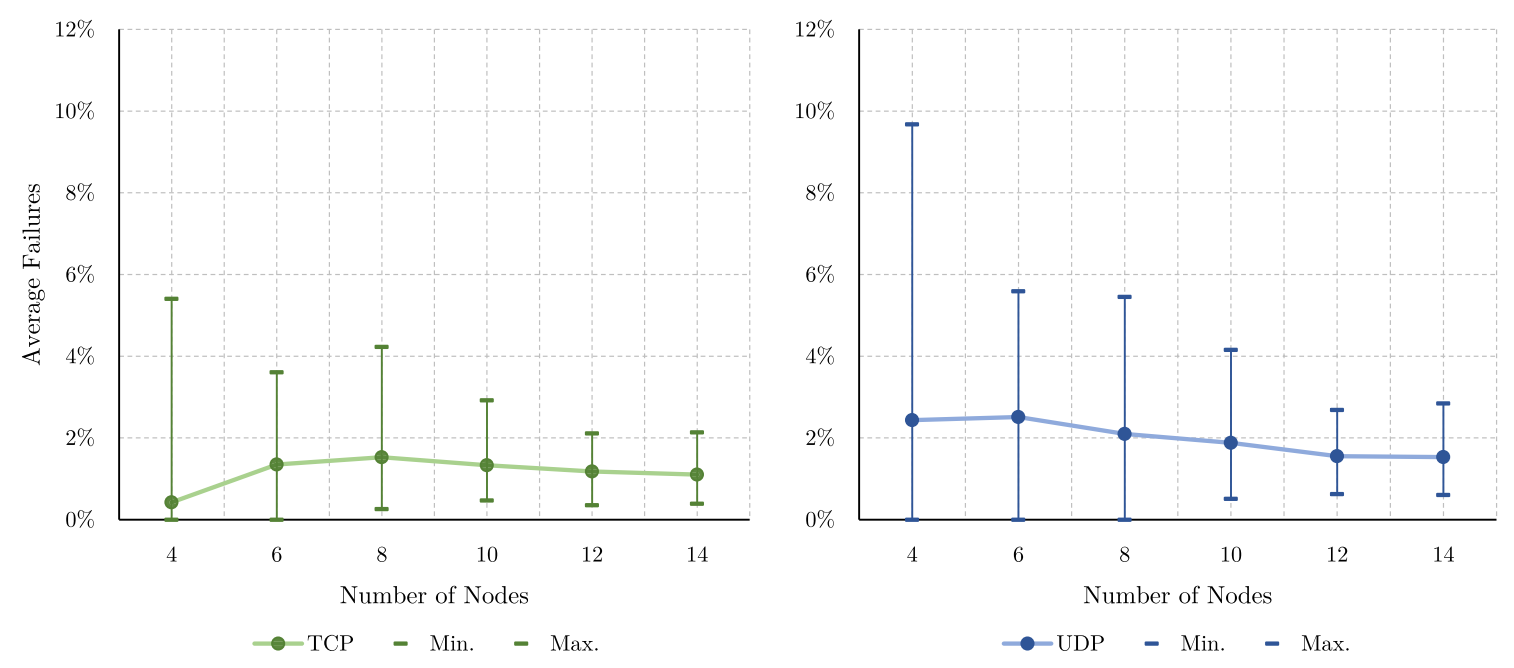

Fig. 7 Average, maximum and minimum percentage of failures in the execution of the host election algorithm according to the total of executions, under TCP and UDP communication protocols, in networks from 4 to 14 nodes

Table 2 Average, maximum and minimum percentage of failures in the execution of the host election algorithm according to the total of executions

\begin{tabular}{llllllll}
\hline \multirow{2}{*}{ Nodes } & UDP & \multicolumn{7}{l}{ TCP } \\
\cline { 2 - 3 } & Average (\%) & Min. (\%) & Max. (\%) & & Average (\%) & Min. (\%) & Max. (\%) \\
\hline 4 & 0.43 & 0.00 & 5.41 & & 2.44 & 0.00 & 9.68 \\
6 & 1.35 & 0.00 & 3.61 & 2.51 & 0.00 & 5.59 \\
8 & 1.53 & 0.26 & 4.23 & & 2.10 & 0.00 & 5.45 \\
10 & 1.33 & 0.47 & 2.92 & & 1.88 & 0.51 & 4.16 \\
12 & 1.18 & 0.36 & 2.11 & & 1.55 & 0.63 & 2.69 \\
14 & 1.10 & 0.39 & 2.14 & & 1.53 & 0.60 & 2.85 \\
Average & 1.15 & - & - & 2.00 & - & - \\
\hline
\end{tabular}

In other words, the election algorithm presents in average a reliability of $98.75 \%$ under TCP and of $98.00 \%$ under UDP.

In respect of the maximum average failures (Table 2), for both protocols, this value is reduced as the network size increments (Fig. 7). Whereas, the minimum average failures is incremented. This is because of the network topology changes increment according the number of nodes increases. Therefore, the network is less stable and the election algorithm is executed more times.

Regarding the reliability provided by the two different transmission protocols, as it was expected, the host election algorithm presents less failures under TCP that under UDP, independently of the network size (Fig. 8). However, the results obtained under TCP highlight the fact that the consensus approach followed by the host election algorithm is not completely reliable on highly dynamic network topologies.

Figure 9 shows the MTBF of the host election algorithm. As occurs with the percentage of failures, the increase of the size of the network leads to an decrease of the MTBF in the execution of the host election algorithm. For small networks, there is a considerable difference between the MTBF given by the host election algorithm under TCP and UDP. However, this difference is reduced as the network grows (Table 3 ). This is due to the exponential consumption of bandwidth of TCP, which begins to saturate the network in the case of ad hoc networks of 12-14 nodes.

The failure in the host election algorithm is usually caused by unstable node groups or links. This failure leads to a situation where there are two services operating at the same time in the same node group, and therefore the self-adaptive architecture does not provide an optimal solution during this time. Usually, these unstable groups disappear in a short period of time, owing to their instability. Figure 10 shows the average, maximum and minimum percentage of wrong operation time of the self-adaptive architecture caused by a failure in the execution of the election algorithm.

Similar to the case of the percentages of failures, under TCP, the self-adaptive architecture presents better operation than UDP (Table 4). While, under TCP the architecture operates correctly on an average $97.24 \%$ of the time, under UDP 


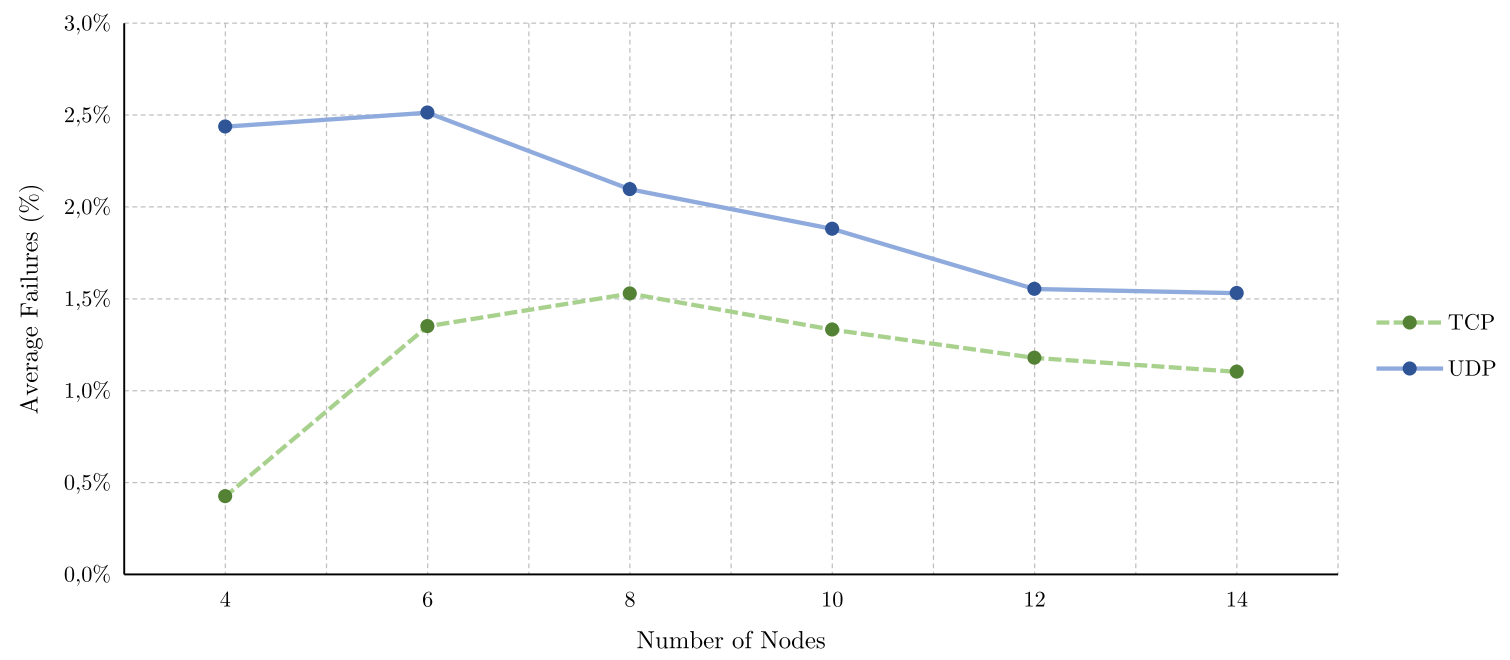

Fig. 8 Average percentage of failures in the execution of the host election algorithm according to the total of executions. TCP communication protocol against UDP

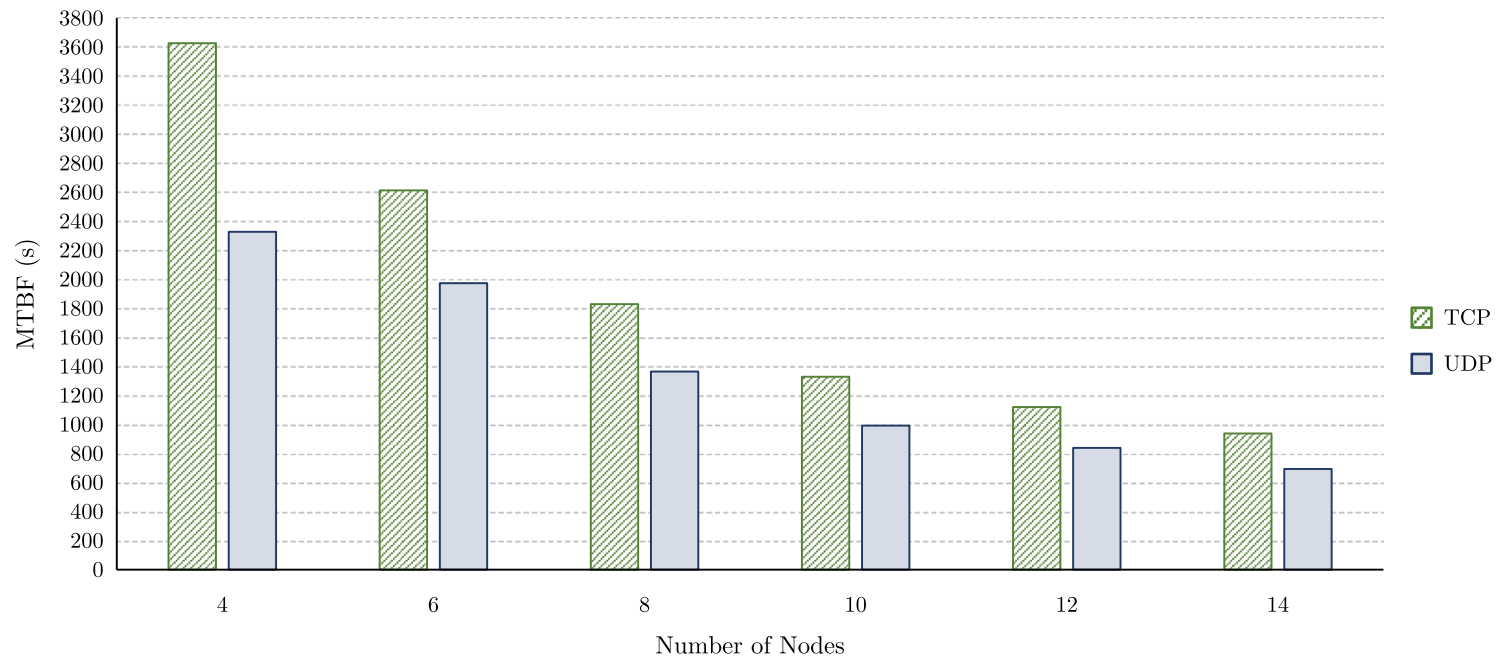

Fig. 9 Mean Time Between Failures (MTBF) of the host election algorithm, under TCP and UDP communication protocols, in networks from 4 to 14 nodes

Table 3 Mean time between failures (MTBF) for the host election algorithm

\begin{tabular}{lrrc}
\hline Nodes & TCP $(\mathrm{s})$ & UDP $(\mathrm{s})$ & Difference $(\mathrm{s})$ \\
\hline 4 & 3627.10 & 2328.12 & 1298.98 \\
6 & 2613.42 & 1973.71 & 639.71 \\
8 & 1829.37 & 1365.06 & 464.31 \\
10 & 1329.26 & 993.13 & 336.13 \\
12 & 1120.58 & 838.97 & 281.62 \\
14 & 938.78 & 694.30 & 244.48 \\
\hline
\end{tabular}

only $96.37 \%$. Likewise, the difference between TCP and UDP regarding the wrong operating time decreases as the size of the network grows (Fig. 11).

\section{Discussion}

Several approaches to address the availability of the services in mobile environments can be found in the literature (3, $5,7,12,18)$. Generally, they are based on adaptive schemes, following the reference model for autonomic control loops, MAPE-K (Monitor, Analyse, Plan, Execute, and Knowledge) (13). However, on one hand, generally, these are ad hoc solutions, which have been developed for specific scenarios, and they are based on an implicit, and often restricted, context model. On the other hand, these works are focused on objectives as load balancing, energy efficiency, scalability, etc. Whereas, the reliability of the system usually is not taken into consideration or is in a second place (23).

From this study, it can be concluded that the reliability of the host election algorithm under TCP presents a bet- 

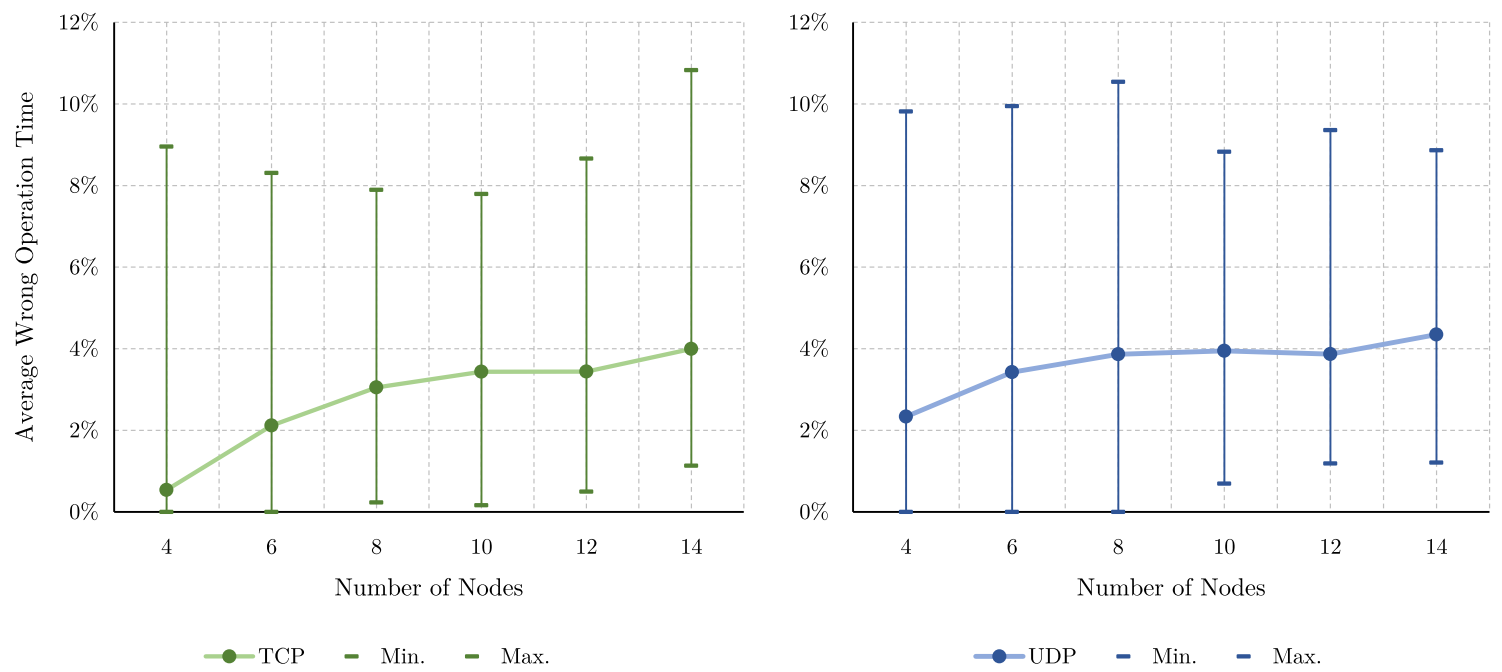

Fig. 10 Average, maximum and minimum percentage of wrong operation time of the self-adaptive architecture according to the total time of execution, under TCP and UDP communication protocols, in networks from 4 to 14 nodes

Table 4 Average, maximum and minimum percentage of wrong operation time of the self-adaptive architecture

\begin{tabular}{|c|c|c|c|c|c|c|}
\hline \multirow[t]{2}{*}{ Nodes } & \multicolumn{3}{|l|}{ TCP } & \multicolumn{3}{|l|}{ UDP } \\
\hline & Average (\%) & Min. (\%) & Max. (\%) & Average (\%) & Min. (\%) & Max. (\%) \\
\hline 4 & 0.54 & 0.00 & 8.95 & 2.34 & 0.00 & 9.82 \\
\hline 6 & 2.12 & 0.00 & 8.31 & 3.42 & 0.00 & 9.95 \\
\hline 8 & 3.05 & 0.23 & 7.89 & 3.87 & 0.00 & 10.54 \\
\hline 10 & 3.44 & 0.16 & 7.79 & 3.95 & 0.69 & 8.83 \\
\hline 12 & 3.44 & 0.50 & 8.66 & 3.87 & 1.19 & 9.36 \\
\hline 14 & 4.00 & 1.13 & 10.83 & 4.35 & 1.21 & 8.87 \\
\hline Average & 2.76 & - & - & 3.63 & - & - \\
\hline
\end{tabular}

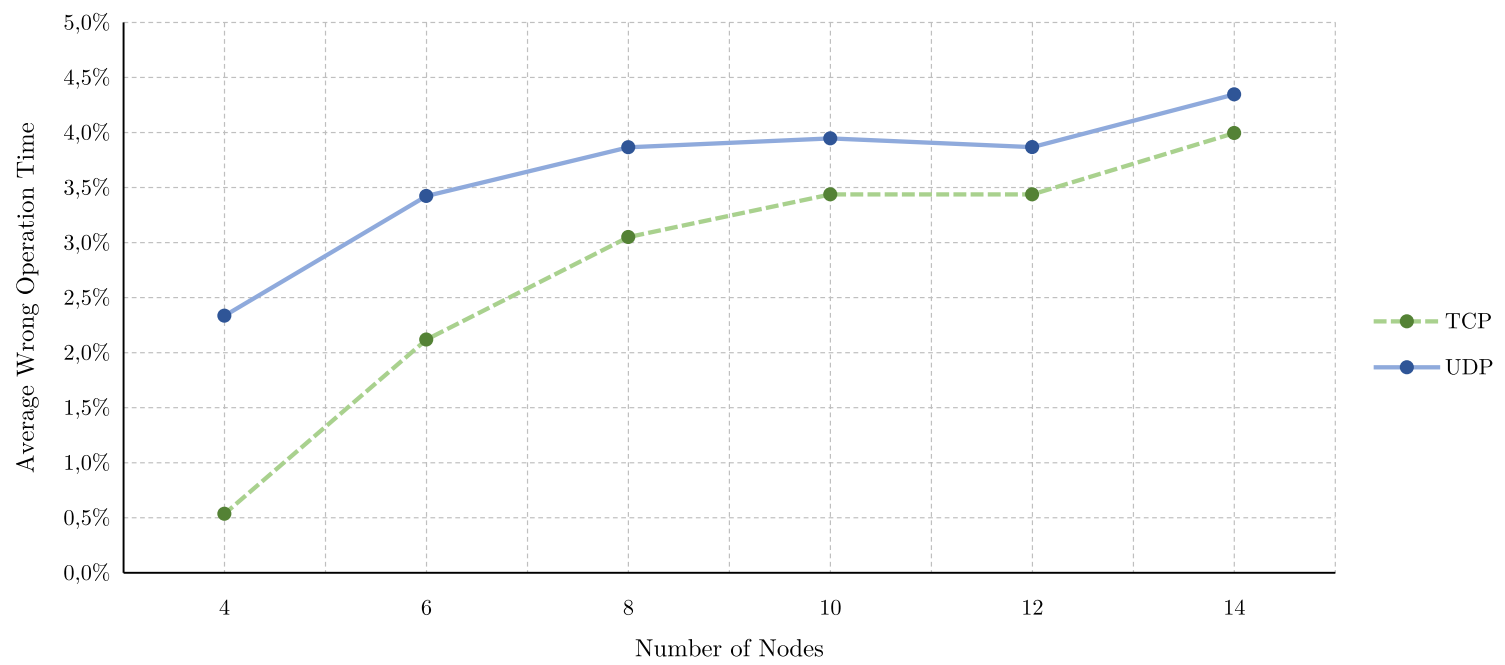

Fig. 11 Average percentage of wrong operation time of the self-adaptive architecture according to the total time of execution. TCP communication protocol against UDP

ter reliability in the message delivery, as it was expected. This is required as the election algorithm follows a consensus approach, and consequently all the nodes within a group must manage the same list of scores. The reliable message delivery could also be implemented in UDP. However, this would be more inefficient than in TCP, among other reasons 
because this task must be implemented and managed in the application layer, instead of in the transportation layer, as TCP does.

Nevertheless, the reliability of the host election algorithm under TCP is close to the provided under UDP for large size networks (98.90\% under TCP, $98.47 \%$ under UDP in a network of 14 nodes). This is caused by the greater requirement of bandwidth of TCP, which begins to saturate the communications in networks of 12-14 nodes. Finally, as the results obtained have shown, the consensus approach followed by the host election algorithm is not completely reliable on highly dynamic network topologies, regardless of the reliability of the communication protocol selected.

\section{Conclusions}

In this work, a self-adaptive architecture (9) and the host election algorithm (11) have been briefly presented in which the architecture is based, as a part of previous work. Additionally, the results obtained from the study have been presented and analysed as to the reliability of the algorithm under different transmission protocols: TCP, a reliable protocol, and UDP, a non-reliable transmission protocol. For this, first, the concept of reliability has been defined and how it is applied to the host election algorithm, defining what is considered as a failure of the algorithm.

Further research is intended to perform a deep study about the behaviour of the host election algorithm, including measures about the response time, usage of bandwidth, message loss, etc. Additionally, some mobility models such as Reference Point Group or Map-based Models will be taken into consideration in order to perform a closer simulation of the system proposed to realistic scenarios. Moreover, it is intended to design and implement a variation of the algorithm, following a voting approach, instead of a consensus approach, in order to release the need of that all the score messages must be delivered to all nodes of the group, providing a more robust approach against message loss. Finally, the current proposal has not addressed the synchronization of replicas of the service. To this regard, the proposal of a general synchronization service is being explored (10). It is based on the combination of cache techniques together an EDA approach to represent and communicate the changes on the shared resources of the network. This service is expected to be included in the current architecture to provide a more complete solution.

Acknowledgements This research is supported by the Centro Mixto Universidad de Granada-MADOC (Spanish Army Training and Doctrine Command), and funded by Banco Santander, under the granted project with Ref. PINS-2014-Cod-9; we thank TCol Doctor Ignacio Alvarez de Cienfuegos (ISFAS Granada) for being part of the project team and for his active help in its progress. This research is also related with the Project Ref. TIN2016-79484-R Granted by the Spanish Ministry of Economy and Competitiveness (FEDER), and the Scholarship Program FPU Ref. FPU13/05520 granted by the Spanish Ministry of Education, Culture and Sports.

\section{References}

Bellavista P, Corradi A, Fanelli M, Foschini L (2012) A survey of context data distribution for mobile ubiquitous systems. ACM Computing Surveys 44(4), pp 1-45. doi:10.1145/2333112.2333119. http://dl.acm.org/citation.cfm?doid=2333112.2333119

Chandrakala CB, Prema KV, Hareesha KS (2012) A Study of Location-Based Data Replication Techniques and Location Services for MANETs Advances in Intelligent Systems and Computing, vol. 167. Springer Berlin Heidelberg. doi:10.1007/ 978-3-642-30111-7

Chandrakala CB, Prema KV, Hareesha KS (2013) Improved data availability and fault tolerance in MANET by replication. In: 2013 3rd IEEE International Advance Computing Conference (IACC) doi:10.1109/IAdCC.2013.6514244

Chlamtac I, Conti M, Liu JJN (2003) Mobile ad hoc networking: imperatives and challenges. Ad Hoc Networks 1:13-64. doi:10.1016/ S1570-8705(03)00013-1

Choudhury P, Sarkar A, Debnath NC (2011) Deployment of Service Oriented architecture in MANET: A research roadmap. In: 20119 th IEEE International Conference on Industrial Informatics doi:10.1109/INDIN.2011.6034957

Coulouris G, Dollimore J, Kindberg T (2012) Distributed Systems: Concepts and Design 5th edn. Addison-Wesley Publishing Company

Derhab A, Badache N (2007) A pull-based service replication protocol in mobile ad hoc networks. Euro Trans Telecomm 18(1):1-11. doi:10.1002/ett.1080

Erl T (2007) SOA principles of service design (Prentice Hall ServiceOriented Computing Series from Thomas ERL)

Guerrero-Contreras G, Balderas-Díaz S, Rodríguez-Domínguez C, Valenzuela A, Garrido J.L (2015) An Approach Addressing Service Availability in Mobile Environments. In: Preuveneers D (ed.) Workshop Proceedings of the 11th International Conference on Intelligent Environments, Prague, Czech Republic, July 1517, 2015, Ambient Intelligence and Smart Environments, vol. 19 doi:10.3233/978-1-61499-530-2-46

Guerrero-Contreras G, Garrido J.L, Balderas-Díaz S, RodríguezDomínguez C (2014) Consistent Management of Context Information in Ubiquitous Systems. In: G. Fortino, G.D. Fatta, W. Li, S.F. Ochoa, A. Cuzzocrea, M. Pathan (eds.) Internet and Distributed Computing Systems - 7th International Conference, (IDCS) 2014, Calabria, Italy, September 22-24, 2014. Proceedings, Lecture Notes in Computer Science, vol. 8729 doi:10.1007/ 978-3-319-11692-1_1

Guerrero-Contreras G, Garrido J.L, Balderas-Diaz S, RodriguezDominguez C (2016) A context-aware architecture supporting service availability in mobile cloud computing. IEEE Transactions on Services Computing PP(99), pp 1-1. doi:10.1109/TSC. 2016.2540629

Hamdy M, Derhab A, König-Ries B: (2010) A Comparison on MANETs Service Replication Schemes: Interest versus Topology Prediction Communications in Computer and Information Science, vol. 84. Springer Berlin Heidelberg, Berlin, Heidelberg. doi:10.1007/978-3-642-14171-3

IBM (2005) Autonomic computing white paper: an architectural blueprint for autonomic computing p 34

Kephart JO, Chess DM (2003) The vision of autonomic computing. Computer 36(1):41-50. doi:10.1109/MC.2003.1160055 
Krill P (2006) Make way for SOA 2.0. InfoWorld http://www. infoworld.com/t/architecture/make-way-soa-20-420

Little M.M, McCue D.D (1994) The Replica Management System: a scheme for flexible and dynamic replication. Proceedings of 2nd International Workshop on Configurable Distributed Systems pp 46-57. doi:10.1109/IWCDS.1994.289936

Mamei M, Zambonelli F, Hutchison D, Mitchell J.C: (2006) SelfManaged Networks, Systems, and Services Lecture Notes in Computer Science, vol. 3996. Springer Berlin Heidelberg, Berlin, Heidelberg. doi:10.1007/11767886. http://www.scopus.com/inward/ record.url?eid=2-s2.0-33746653696\&partnerID $=$ tZOtx3y 1

Psaier H, Juszczyk L, Skopik F, Schall D, Dustdar S (2010) Runtime behavior monitoring and self-adaptation in service-oriented systems. doi:10.1109/SASO.2010.44

Rodríguez-Domínguez C, Benghazi K, Garrido J.L, Garach A.V (2013) Designing a Communication Platform for Ubiquitous Systems: The Case Study of a Mobile Forensic Workspace. In: V.M. Penichet, A. Peñalver, J.A. Gallud (eds.) Human-Computer Interaction Series HumanComputer Interaction Series pp. 97-111. Springer London, London. doi:10.1007/978-1-4471-5445-7_8
Rodríguez-Domínguez C, Benghazi K, Noguera M, Garrido JL, Rodríguez ML, Ruiz-López T (2012) A Communication model to integrate the Request-Response and the publish-subscribe paradigms into ubiquitous systems. Sensors (Switzerland) 12(6):7648-7668. doi:10.3390/s120607648

Tortorella M: (2015) Reliability, maintainability, and supportability: best practices for systems engineers. Wiley

Weyns D, Ahmad T (2013) Claims and evidence for architecture-based self-adaptation: A systematic literature review. Lecture Notes in Computer Science (including subseries Lecture Notes in Artificial Intelligence and Lecture Notes in Bioinformatics) 7957 LNCS, pp 249-265. doi:10.1007/978-3-642-39031-9_22

Xu L, O'Grady M, O’Hare G, Collier R (2014) Reliable multihop intra-cluster communication for Wireless Sensor Networks. 2014 International Conference on Computing, Networking and Communications (ICNC) pp. 858-863. doi:10.1109/ICCNC.2014. 6785450 